\title{
Recursos didácticos y tecnológicos para la enseñanza de la integral definida en el modelo de Universidad Comunitaria Intercultural
}

William Oswaldo Flores López ${ }^{1}$

Eugenio López Mairena ${ }^{2}$

\section{Resumen}

Esta investigación ha descrito los recursos didácticos y tecnológicos que el profesorado de matemáticas incorpora en el proceso de enseñanza de la integral definida, la evaluación que el profesorado efectúa, y las dificultades de comprensión en el aprendizaje del estudiantado. Fue un estudio cuantitativo y cualitativo sustentado en un estudio de caso, con una muestra de 46 estudiantes del tercer semestre de Administración de Empresas y 12 profesores de Matemáticas de la Universidad de las Regiones Autónomas de la Costa Caribe Nicaragüense, Recinto Universitario Nueva Guinea. El análisis se desarrolló con énfasis en las capacidades observadas en el aula de clases, y las argumentaciones en respuesta a los cuestionarios y entrevistas dirigidas al estudiantado y el profesorado. Los resultados sostienen que la incorporación de recursos didácticos y tecnológicos en la enseñanza de la integral definida disminuye las dificultades de comprensión en el aprendizaje, lo que se traduce en que al diseñar e implementar recursos didácticos y tecnológicos, se convierte en un entorno de agrado, motivación y confianza para que el estudiantado resuelva tareas matemáticas, y por tanto, disminuye sus dificultades de aprendizaje.

Palabras clave: Recursos didácticos; recursos tecnológicos; enseñanza-aprendizaje; integral definida; actitudes hacia las matemáticas.

\section{Summary}

This research has described the educational and technological resources that the math teachers incorporates in the process of teaching the definite integral, the evaluation made by the teachers, and the difficulties the students presents in its understanding. It was a quantitative and qualitative study based on a case study, with a sample of 46 students from the third semester of Business Administration and 12 math teachers from the University of the Autonomous Regions of the Caribbean Coast of Nicaragua, Nueva Guinea campus. The analysis was developed with emphasis on the capacities

1 Doctor en Educación, Profesor, Investigador y Gestor de Proyectos de la Universidad de las Regiones Autónomas de la Costa Caribe Nicaragüense, Nicaragua. Email: william.flores@uraccan.edu.ni

2 Doctor en Innovación en la Formación, Vicerrector de la Universidad de las Regiones Autónomas de la Costa Caribe Nicaragüense, Recinto Universitario Nueva Guinea. Email: vicerectoria.ng@uraccan.edu.ni 
observed in the classroom, and the arguments in response to the questionnaires and interviews aimed to students and teaching staff.

The results demonstrate that the incorporation of educational and technological resources in the teaching of definite integral reduces the difficulties for a comprehensive learning, which means that when designing and implementing educational and technological resources it can create a pleasing environment full of motivation and confidence in order that the students resolve their mathematical tasks, and therefore decreases their learning difficulties.

Keywords: Educational resources; technological resources; teaching and learning; definite integral; attitudes toward mathematics.

\section{Introducción}

Históricamente la enseñanza y aprendizaje del cálculo diferencial e integral no han producido los resultados deseados en la formación educativa del estudiantado de Educación Superior. En la actualidad, esta situación no ha cambiado y se refleja en el desempeño del estudiantado, quienes al ingresar a la universidad manifiestan un deficiente aprendizaje, con nociones pobres acerca de las matemáticas elementales, exhibiendo un cúmulo de ideas confusas que difícilmente asimilan o retienen, provocando dificultades en la comprensión de nociones y conceptos básicos de los pensamientos: algebraico, geométrico y estadístico. Estas situaciones pueden tener importantes repercusiones cuando el estudiantado se enfrenta con asignaturas de matemáticas cuyos contenidos tienen un mayor nivel de complejidad cognitiva y que pueden tener mucha importancia en su desarrollo profesional.

En lo que respecta a la enseñanza y aprendizaje del concepto-propiedades de la integral definida, en la Universidad Comunitaria Intercultural se aborda en las especialidades vinculadas a las áreas de: Ciencias Administrativas e Informáticas, Tecnología de la Construcción, Recursos Naturales y Ciencias de la Educación, específicamente en los grados de matemáticas y física-matemática. En este sentido, la enseñanza de la integral definida es impartida en función de tres dimensiones: el contenido al que se refieren los problemas matemáticos; los procesos que deben activarse para conectar los fenómenos observados con las matemáticas y resolver así problemas correspondientes; y, por último las situaciones y los contextos utilizados como fuentes de recursos didácticos y tecnológicos en los que se plantean los problemas.

Por lo tanto, esta investigación se centra en describir los recursos didácticos y tecnológicos que el profesorado de matemáticas incorpora en el proceso de enseñanza de la integral definida y, a su vez, el proceso de evaluación que el profesorado efectúa, lo que permitirá categorizar las dificultades de comprensión en el aprendizaje del estudiantado. Todo ello, con la premisa que los recursos didácticos y tecnológicos 
que incorpora el profesorado de matemáticas en la enseñanza de la integral definida disminuye las dificultades de comprensión en el aprendizaje del estudiantado.

\section{Revisión de literatura}

En la enseñanza y aprendizaje de las matemáticas, los recursos didácticos y tecnológicos juegan un papel importante en la construcción del conocimiento matemático de las poblaciones en contexto de diversidad. Sin embargo, no basta con incorporar los recursos didácticos y tecnológicos en el aula de clases, antes es necesario reconocer la particularidades de las poblaciones productoras y usuarias de tales recursos (León et al., 2013). Para esto se hace indispensable generar estrategias para dinamizar la relación que los individuos establecen entre sí y con las comunidades a las que pertenecen; éstas aportan diferentes elementos a los individuos y a los tipos de prácticas que les permite construir su identidad (León et al., 2014). Así, Flores (2015a) afirma que una metodología elegida para la formación matemática es la perspectiva de comunidades de aprendizajes, en términos teórico la comunidad de aprendizaje comprende todas las actividades orientadas a la creación, recreación, diseminación e intercambio de conocimientos, saberes, valores y prácticas desde dos vías: la interacción entre conocimientos locales y ancestrales y conocimientos occidentales para el desarrollo de competencias en el mundo del trabajo, el desarrollo de valores y actitudes que preparen a los educandos para enfrentar con éxito los desafíos de la vida (URACCAN, 2014).

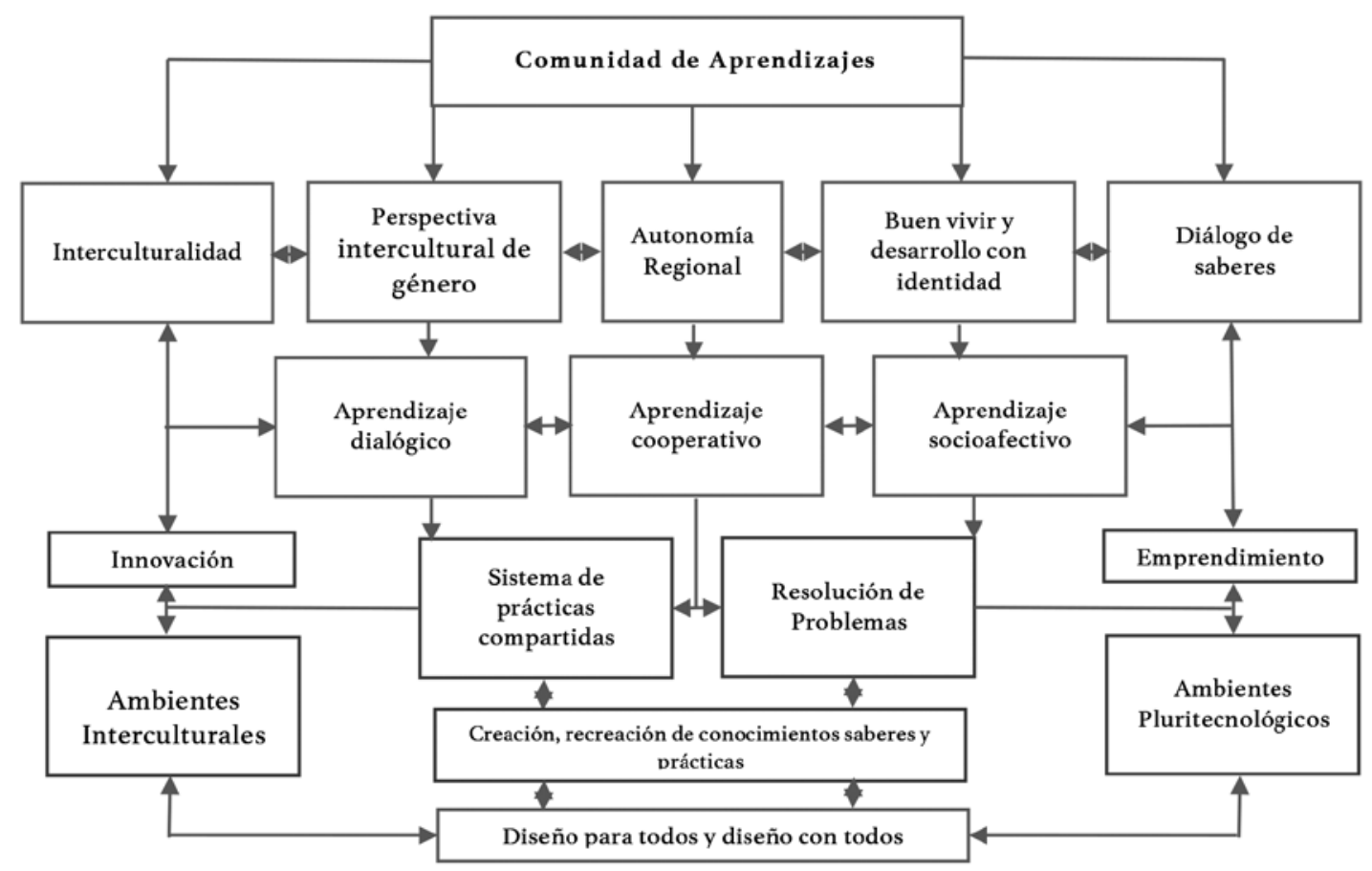

Figura No. 1: Modelo de enseñanza de las matemáticas. Fuente: Flores (2016). 
Desde esta perspectiva, se orientará el procesos de enseñanza-aprendizaje utilizando el enfoque intercultural, el cual se pronuncia por el desarrollo de un diálogo intercultural como una estrategia para promover espacio de innovación de nuevos conocimientos, ya que confronta elementos de diferentes horizontes y perspectivas culturales, abriendo la posibilidad de impulsar un proceso de complementación y enriquecimiento entre la ciencia moderna y otros saberes (URACCAN, 2014). Así, la formación del estudiantado en contextos de diversidad se configura como un proceso de negociabilidad de lo matemático en una comunidad de práctica (León et al., 2013) en la que los aspectos siguientes se manifiestan y se tematizan: interculturalidad, perspectiva intercultural de género; autonomía regional, buen vivir y desarrollo con identidad; y diálogo de los saberes (Flores, 2015a).

En esta dirección, la metodología se articula a partir de los fundamentos teóricos que ofrecen las pedagogías iluminadas por el enfoque histórico cultural. Por tal razón, se considera valioso orientar la formación hacia aspectos duales tales como: participación-cosificación, local y global, negociabilidad de significados-identificación, emergencia-diseño (Gil et al., 2013). En este sentido, es necesario que el profesorado produzca sus recursos didácticos y tecnológicos (Diseño para todos y diseño con todos) para que el estudiantado en cualquier condición sensorial, lingüística, cultural o socioeconómica interactuando juntos aprendan matemáticas y así el estudiantado se forma en ambiente de coexistencia con la diversidad de poblaciones (León et al., 2013). También, hay que tomar en cuenta que, en los recursos didácticos y tecnológicos se incorpora un sistema de prácticas compartidas, que se definen como el conjunto de acciones asociadas al hacer matemáticas, es decir, formular, probar, construir modelos, lenguajes, conceptos, teorías, intercambiar construcciones con otros y reconocer construcciones útiles a prácticas matemáticas en cada cultura (León et al., 2014).

Referente con la evaluación en la enseñanza de las matemáticas en el modelo de Universidad Comunidad Intercultural es asumida desde la siguiente perspectiva:

- La importancia de la evaluación radica en el acercamiento del individuo a las normas y pautas culturales que permiten salvaguardar la identidad del pueblo.

- Una característica de la evaluación para poblaciones indígenas, afrodescendientes y mestiza, es que se da la relación estrecha de padre-hijo, madre-hija, y otros parientes en el momento mismo en el que el hijo o la hija realiza determinada actividad. Se evalúa diariamente.

- La evaluación para este tipo de poblaciones también se caracteriza por su carácter cualitativo y es evaluación integral porque se consideran todos los ámbitos de la vida del estudiantado: afectivo cognoscitivo y psicomotor.

- El aspecto valorativo en la evaluación para este contexto diverso es una responsabilidad colectiva, porque participan los miembros de la comunidad, tanto mayores como grupo de igual edad. 
La evaluación de los aprendizajes es asumida por la estrategia de comunidades de práctica (Wenger, 2001), se constituyen si entre sus miembros existe un compromiso mutuo de realizar una cierta empresa, lo que lleva a una práctica compartida o sistemas de prácticas compartidas en la que se genera aprendizaje compartido y significativo. Por lo tanto, para evaluar o resolver un problema matemático se promueve un aprendizaje dialógico que configure la discusión y argumentación entre la interacción estudiante-estudiante y estudiante-profesor (Coll y Colomina, 1991); un aprendizaje colaborativo que ayude a representar, plantear, modelar y resolver problemas; y un aprendizaje socio afectivo que ayude a crear un ambiente comunicativo e interactuar entre estudiante-estudiante y estudiante-profesor para dar respuesta a la tarea o problema matemático.

\section{Materiales y métodos}

Esta investigación integra los métodos cuantitativos y cualitativos con el fin de interpretar la situación vital en la que los sujetos han experimentado el fenómeno que se quiere investigar y así estudiar las semejanzas y diferencias que hay entre dos situaciones, con el propósito de describir los factores que parecen explicar la presencia del fenómeno en una situación y su ausencia en la otra (Stake, 1999). En este sentido, se sustenta en un estudio de caso donde se describen los recursos didácticos y tecnológicos de evaluación en el aprendizaje de la integral definida.

El estudio se efectuó con 46 estudiantes universitarios del tercer semestre en Administración de Empresa de la URACCAN, Recinto Nueva Guinea, pertenecientes a comunidades de la Costa Caribe de Nicaragua. La selección de los participantes se realizó mediante un muestro por conveniencia, es decir, por accesibilidad y proximidad de los sujetos, al investigador. La edad media entre los participantes fue de 19 años con un rango comprendido entre los 18 y los 21 años. El 62\% de los participantes fueron mujeres y el $38 \%$ restante fueron hombres. En el caso del profesorado fueron 12 profesores de matemáticas, el $16 \%$ del profesorado fueron mujeres y el $84 \%$ fueron hombres, con una intervalo entre 7 a 10 años de experiencia académica.

Para recopilar la información se aplicó un test cognitivo y cuestionario escala Likert al estudiantado, una entrevista al profesorado, y observaciones participantes y no participantes a las clases del profesorado de matemáticas. La reconstrucción y descripción del estudio se logró gracias a las tipologías de análisis, entre ellos: el análisis referido a la entrevista y la observación se desarrolló a partir de una metodología basada en criterios donde se incluyen aspectos cualitativos y cuantitativos de las respuestas. En el análisis del cuestionario tipo escala de Likert se realizó un análisis descriptivo a través del Análisis Estadístico utilizando el Software Estadístico para las Ciencias Sociales (SPSS). Mientras que el cuestionario cognitivo se basan en el análisis cualitativo de las respuestas, a través de la categorización de las mismas, poniendo como énfasis las capacidades observadas de argumentación de las respuestas. 
Las conclusiones y discusión se articulan a través de la idoneidad didáctica basada en el enfoque ontológico y semiótico del conocimiento y de la instrucción matemática (Godino, 2013).

\section{Resultados y discusión}

\section{Recursos didácticos y tecnológicos en el aprendizaje de la integral definida}

Los recursos didácticos que el profesorado de matemáticas utiliza se enfocan en las tipologías: (i) El currículo académico de la espacialidad, programas de la asignatura y libros de textos; (ii) Plataforma virtual Moodle; (iii) Software matemáticos; y (iv) Dispositivos tecnológicos. Estos se transforman en materiales didácticos que se aplican en diferentes contextos. Véase el Cuadro No. 1.

Cuadro No. 1. Recursos didácticos en el aprendizaje de la integral definida

\begin{tabular}{|l|l|l|}
\hline \multicolumn{1}{|c|}{ Recursos didácticos y tecnológicos } & \multicolumn{1}{|c|}{ Materiales didácticos y tecnológicos } & \multicolumn{1}{c|}{ Contexto de aplicación } \\
\hline $\begin{array}{l}\text { Currículo académico de las } \\
\text { especialidades, programas } \\
\text { de la asignatura y libros de } \\
\text { texto. }\end{array}$ & $\begin{array}{l}\text { Syllabus, unidades didácti- } \\
\text { cas, guías de aprendizajes. }\end{array}$ & $\begin{array}{l}\text { Situaciones didácticas en el } \\
\text { contexto social y cultural del } \\
\text { estudiantado. }\end{array}$ \\
\hline Plataforma virtual Moodle. & $\begin{array}{l}\text { Objetos virtuales de apren- } \\
\text { dizaje. }\end{array}$ & $\begin{array}{l}\text { Power Point, Excel, videos, } \\
\text { pizarra interactiva, periódi- } \\
\text { cos (economía), mundos } \\
\text { virtuales. }\end{array}$ \\
\hline Software matemático. & $\begin{array}{l}\text { Modelización de las situacio- } \\
\text { nes matemáticas. }\end{array}$ & $\begin{array}{l}\text { Costo marginal, ingreso mar- } \\
\text { ginal, función oferta, función } \\
\text { demanda, superávit de con- } \\
\text { sumidores y productores, } \\
\text { inventario diario promedio, } \\
\text { el valor presente de un flujo } \\
\text { de ingreso. }\end{array}$ \\
\hline Dispositivos tecnológicos. & $\begin{array}{l}\text { Calculadoras, ordenadores y } \\
\text { celulares. }\end{array}$ & $\begin{array}{l}\text { Simulación del cálculo alge- } \\
\text { braico simbólico y cálculo } \\
\text { interactivo geométrico. }\end{array}$ \\
\hline
\end{tabular}

Estos recursos didácticos y tecnológicos que el profesorado diseña e implementa para las clases de matemáticas, lo organizan en campos estructurantes como personalfamiliar, social-comunitario, profesional-especializado, científico-tecnológico (Goñi, 2008), particularmente: 
- Personal-familiar: relacionado con las actividades diarias, y en el que el estudiantado debe activar su conocimientos matemáticos para interpretar los aspectos relevantes de situaciones cotidianas.

- Social-comunitario: se refiere a situaciones donde el estudiando debe relacionar diversos elementos del entorno social o entorno comunitario, para evaluar qué aspectos del mismo tienen consecuencias relevantes.

- Profesional-especializado: referido a situaciones que surgen en la universidad o trabajo relacionada con su especialización con problemas que requieren una solución matemática.

- Científico-tecnológico: incluye contenidos más abstractos como la comprensión de procesos tecnológicos o la explicación de problemas matemáticos. Esta categoría abarca también situaciones del cálculo diferencial e integral abstracto que pueden surgir en las clases, y que requieren explicitar los elementos matemáticos del problema para situarlo en contexto más amplio.

En este sentido, el profesorado de matemática planifica la enseñanza de la integral definida, teniendo en cuenta aspectos como: la definición del enfoque didáctico; definición de múltiples experiencias -prácticas, ficticias, hipotéticas-; definición de múltiples ambientes de aprendizaje; definición de ambientes de aprendizaje para la diversidad; definición de la utilización de recursos tecnológicos con participación activa y propositiva; definición del propósito de las matemáticas en la especialidad; y que los recursos didácticos estén acorde al planteamiento de la temática. Todo ello, con una tendencia positiva entre excelente a bueno (con un promedio del $77 \%$ ).

Cuadro No. 2. Elementos que incorporan el profesorado en la planificación de la enseñanza

\begin{tabular}{|l|l|l|l|l|l|}
\hline $\begin{array}{c}\text { Elementos para la } \\
\text { planificación de la } \\
\text { enseñanza }\end{array}$ & Excelente & Muy Bueno & Buena & Regular & Deficiente \\
\hline $\begin{array}{l}\text { Define el enfoque } \\
\text { didáctico. }\end{array}$ & $31 \%$ & $29 \%$ & $29 \%$ & $6 \%$ & $5 \%$ \\
\hline $\begin{array}{l}\text { Define múltiples ex- } \\
\text { periencias -prácticas, } \\
\text { ficticias, hipotéticas. }\end{array}$ & $18 \%$ & $27 \%$ & $22 \%$ & $20 \%$ & $13 \%$ \\
\hline $\begin{array}{l}\text { Define múltiples siste- } \\
\text { ma de representación. }\end{array}$ & $18 \%$ & $30 \%$ & $30 \%$ & $16 \%$ & $6 \%$ \\
\hline $\begin{array}{l}\text { Define múltiples } \\
\text { ambientes de apren- } \\
\text { dizaje. }\end{array}$ & $17 \%$ & $30 \%$ & $20 \%$ & $18 \%$ & $14 \%$ \\
\hline $\begin{array}{l}\text { Define ambientes de } \\
\text { aprendizaje para la } \\
\text { diversidad. }\end{array}$ & $30 \%$ & $28 \%$ & $17 \%$ & $12 \%$ & $13 \%$ \\
\hline
\end{tabular}




\begin{tabular}{|l|l|l|l|l|l|}
\hline $\begin{array}{c}\text { Elementos para la } \\
\text { planificación de la } \\
\text { enseñanza }\end{array}$ & Excelente & Muy Bueno & Buena & Regular & Deficiente \\
\hline $\begin{array}{l}\text { Define la utilización } \\
\text { de recursos tecnológi- } \\
\text { cos con participación } \\
\text { activa y propositiva. }\end{array}$ & $33 \%$ & $16 \%$ & $22 \%$ & $13 \%$ & $17 \%$ \\
\hline $\begin{array}{l}\text { Define el propósito } \\
\text { de la matemática en } \\
\text { la especialidad. }\end{array}$ & $27 \%$ & $34 \%$ & $30 \%$ & $8 \%$ & $1 \%$ \\
\hline $\begin{array}{l}\text { Los recursos didácti- } \\
\text { cos están de acorde a } \\
\text { lo planteamientos de } \\
\text { la temática. }\end{array}$ & $29 \%$ & $22 \%$ & $34 \%$ & $10 \%$ & $6 \%$ \\
\hline $\begin{array}{l}\text { Promedio en las pun- } \\
\text { tuaciones. }\end{array}$ & $25 \%$ & $27 \%$ & $25 \%$ & $13 \%$ & $9 \%$ \\
\hline
\end{tabular}

Algo importante a destacar, en la enseñanza de la integral definida es la incorporación de la plataforma virtual Moodle, la cual integra recursos tecnológicos accesible para el estudiantado (Flores \& Rojas, 2013). Es decir, el curso se organiza a través de un conjunto de bloques de contenidos, para cada de los cuales se facilita el desarrollo del tema correspondiente, así como un conjunto de materiales complementarios a los tópicos descritos en dicho bloque. Puesto que se trata de un curso muy práctico dirigido a presentar y adquirir una serie de competencias en el desarrollo de la integral definida, a su vez, se facilitan diversos videos tutoriales para agilizar la implementación y el estudio de cada bloque temático.

Así mismo, cada bloque de contenido incluye un conjunto de actividades y tareas que son necesarias realizar para superar el curso. Las actividades se diseñan según el enfoque metodológico que se promueve en el apartado de recursos didácticos y son auto-contenidas. El objetivo fundamental de utilizar recursos tecnológicos en el aprendizaje de la integral definida es construir un ambiente pluritecnológico que desarrolle los saberes de los estudiantado, configurado a sus necesidades tecnológicas y facilite la resolución de las dudas y la colaboración en un entorno integrado de enseñanza-aprendizaje. En este sentido, el proceso formativo de la integral definida consisten en una serie de mecanismo de comunicación profesor-estudiante, entre los que se destacan la creación de foros, tantos genéricos (dudas y comentarios acerca del curso y su seguimiento) como específicos por bloques temáticos o tópicos especiales de interés para el curso. Además, el profesorado de matemáticas incorpora entornos informáticos como Maple, Matlab, Wrmaxima, Derive, Mathematics, GeoGebra, Excel y dispositivos tecnológicos como celulares con aplicaciones abiertas para calcular y graficar la integral definida, así también calculadoras gráficas. 
Con respecto a lo anterior, el estudiantado realiza las actividades de forma individual o colaborativa, y posteriormente envía su solución-resolución al profesorado del curso mediante los canales de control de tareas-foros interno del propio entorno virtual educativo. En este entorno virtual quedan reflejadas las fechas de entrega, fecha de evaluación, la valoración de las mismas y los comentarios de retroalimentación de cada una de las actividades.

\section{Proceso de evaluación de la integral definida}

La evaluación en el aprendizaje de la integral definida es asumida desde la perspectiva de comunidad de práctica, el profesorado de matemáticas promueve: estimulación para la participación, dominio del grupo, dominio de la disciplina, instrumentos de evaluación, estilos metodológicos y la planificación adecuada.

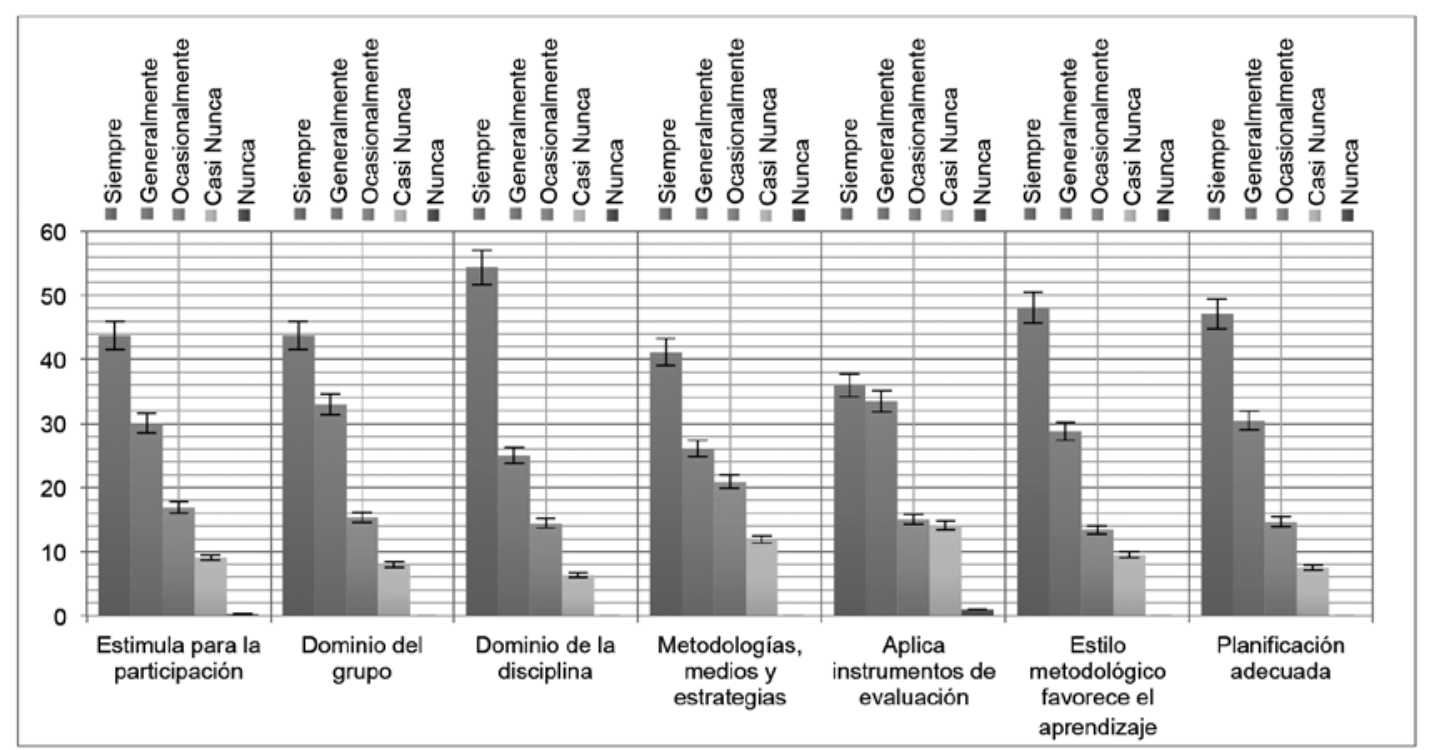

Figura No. 2. Proceso de evaluación en el aprendizaje de la integral definida

Los elementos de evaluación considerados por los profesores son la evaluación diagnóstica puntual al inicio, durante y al finalizar sus clases, como un referente para lograr los objetivos propuestos. Registrando los pre saberes de los estudiantes, al hacer preguntas claves o generadoras de conceptos e ideas varias sobre un determinado tema o interrogante de una tarea asignada. Así mismo el profesorado toma en cuenta los resultados de la evaluación diagnóstica; presentando algunas alternativas o medidas para mejorar el aprendizaje del estudiantado, facilitando la comprensión del tema de clase a desarrollar.

En el diagnóstico el profesorado retoma la participación de los estudiante en el transcurso de los diferentes momentos del aprendizaje, se flexibiliza a partir de su 
percepción, toma en cuenta que el tiempo y posibles dificultades del mismo, se consideran los factores que son útiles antes de pasar al nuevo tema o esclarecer posibles dudas, es válido que exista una correspondencia y pertinencia de las fases de la diagnosis durante el transcurso de la etapa del proceso de aprendizaje. Con referencia al aspecto cualitativo de la evaluación, los profesores afirman que hoy en día el carácter innovador es imprescindible en todo proceso de enseñanza- aprendizaje, y que el profesorado, además de ser facilitador del conocimiento, debe valorar la participación y desempeño del estudiantado en el aula de clase, de aquí se deduce que el profesorado evalúa a través de la observación llevando un registro de participación o desempeño.

La particularidad de la forma de evaluación que se aplica en el proceso de instrucción permite al profesorado contrastar los resultados de las experiencias de aprendizaje con el desempeño personal, valorando su esfuerzo, cumplimiento y calidad de las asignaciones. En síntesis, el profesorado durante el desarrollo de una actividad, plantean situaciones de choques cognitivos entre los estudiantes para tomar en cuenta los aspectos cognoscitivos, procedimentales y actitudinales. Destacamos que el trabajo constructivista de los aprendizajes en comunidad de prácticas se realiza a partir de los grupos interactivos con énfasis en el reconocimiento a la diversidad de los participantes y una comunicación horizontal. Los resultados de esta experiencia son formativos y tendrán influencia en la toma de decisiones en el aula.

\section{Dificultades de comprensión en el aprendizaje de la integral definida}

Las dificultades de comprensión del estudiantado en el aprendizaje de la integral definida se clasifican en: dificultades asociadas al álgebra con un $27 \%$, obstáculos cognitivos con un $35 \%$, errores en el proceso de generalización con un $38 \%$. Las podemos observar en el Cuadro No. 3.

Cuadro No. 3. Dificultades en el aprendizaje de la integral definida

\begin{tabular}{|c|c|c|c|}
\hline No & $\begin{array}{l}\text { Dificultades en el aprendizaje } \\
\text { de la integral definida }\end{array}$ & Errores comunes & $\%$ \\
\hline \multirow{3}{*}{1.} & \multirow{3}{*}{ Dificultades asociadas al álgebra. } & Errores por el uso y dominio de las letras. & \multirow{3}{*}{27} \\
\hline & & Errores por el uso y dominio del signo igual. & \\
\hline & & Necesidades de particularizar expresiones. & \\
\hline \multirow{2}{*}{2.} & \multirow{2}{*}{$\begin{array}{l}\text { Dificultades por un obstáculo } \\
\text { cognitivo. }\end{array}$} & $\begin{array}{l}\text { Origen en el concepto de función, series, su- } \\
\text { cesiones, desigualdades o inecuaciones. }\end{array}$ & \multirow{2}{*}{35} \\
\hline & & $\begin{array}{l}\text { Origen en el concepto de límite, derivada e } \\
\text { integral no definida. }\end{array}$ & \\
\hline
\end{tabular}




\begin{tabular}{|c|l|l|c|}
\hline No & $\begin{array}{l}\text { Dificultades en el aprendizaje } \\
\text { de la integral definida }\end{array}$ & \multicolumn{1}{|c|}{ Errores comunes } & \% \\
\hline \multirow{3}{*}{3.} & \multirow{2}{*}{$\begin{array}{l}\text { Errores en el proceso de genera- } \\
\text { lización. }\end{array}$} & $\begin{array}{l}\text { Errores de distinguir lo particular para llegar } \\
\text { a una conclusión concreta. }\end{array}$ & $\begin{array}{l}\text { Errores en la deducción de métodos y reglas } \\
\text { generales. }\end{array}$ \\
\cline { 3 - 3 } & & $\begin{array}{l}\text { Errores en los sistemas de registros de repre- } \\
\text { sentación semiótico. }\end{array}$ & \\
\hline
\end{tabular}

Las dificultades asociadas al álgebra son por la naturaleza del lenguaje algebraico en el contexto matemático, lo que conlleva al estudiantes a cometer errores por el uso y dominio de las letras (Flores, 2015b), errores por el uso y dominio del signo igual (Molina et al., 2009) y necesidades de particularización de expresiones algebraico al lenguaje numérico (Flores, 2016). Referente a los obstáculos estos son conocimientos adquiridos, no una falta de conocimiento (González-Martín \& Camacho, 2005) y se manifiestan en la etapa de apropiación del conocimiento de función, serie y sucesiones, desigualdades o inecuaciones, límite, derivada e integral no definida (Flores \& López, 2010). Los errores en el proceso de generalización son de carácter de distinguir lo particular para llegar una conclusión concreta (Alonso et al., 1993), errores en la deducción de método y reglas generales (González-Martín, 2002), y errores en los registro de representación semiótica (Duval, 1995).

Otro aspecto a señalar, son las emociones-actitudes-creencias relacionadas con la utilidad, confianza y motivación en el sentido de la comunicación e interacción en el aula (Flores \& Auzmendi, 2015). Esta apreciación se observa en la figura siguiente.

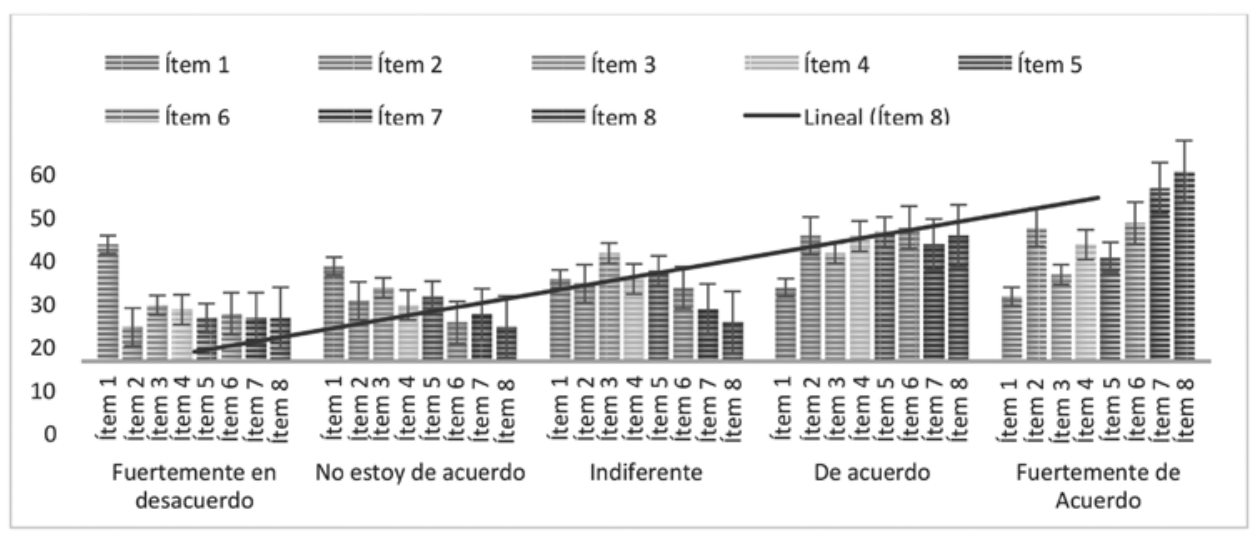

Figura No. 3. Actitudes hacia aprender la integral definida en la especialidad

La figura No. 3, se observa en el ítem No. 1, que el 32\% del estudiantado considera que el contenido de la integral definida no es muy necesaria en sus estudios; mientras que, en el ítem 2, el 60\% de los estudiantes creen que el contenido de la 
integral definida le servirá en su vida futura, estas dos tipologías de actitudes se refleja en el ítem 3, donde el $45 \%$ de los estudiantes afirman conocer la utilidad de la integral definida en su especialidad, sabiendo que, a nivel universitario el papel afectivo hacia las matemáticas juega un papel importante, es decir, la utilidad hacia el aprendizaje de la integral definida juega un papel significativo en la construcción de nuevos conocimientos (Flores \& López 2010). Por otra parte, la falta de motivación por el estudiantado se manifiesta en un ambiente de desconfianza en la línea de no trabajar aplicaciones en el contexto sociocultural y aplicaciones en el contexto de la especialidad de los estudiantes (Flores \& Salinas, 2013).

Lo anterior se puede interpretar en dos líneas, la primera: en el ítem 4, el 56\% del estudiantado valoran positiva las explicaciones del profesorado trasmitiendo que les da confianza para resolver problemas y, a su vez, en el ítem 5 , el 54\% del estudiantado le gusta realizar las prácticas matemáticas asignadas por el profesorado. En concreto, las explicaciones proporcionan confianza y seguridad para resolver una tarea matemática (Cardoso, Vanegas \& Cerecedo, 2012). La segunda línea: en el ítem 6, el 63\% del estudiantado le motiva interactuar con los recursos didácticos que usa el profesor para la enseñanza, así como, el $67 \%$ del estudiantado, le motiva interactuar con los recursos tecnológicos (ítem 7). Todo ello, conlleva, a que, el 73\% del estudiantado se sienta motivado en aprender conceptos y propiedades de la integral definida en su especialidad (ítem 8).

En conclusión, resulta trascendental que los profesores de matemáticas sean conscientes de cómo la reacción emocional en el aprendizaje de las matemáticas puede estar ligada a la comunicación e interacción en el aula, a la interacción social y al contexto cultural (Mandler, 1989). Por ello, el diseño y construcción de una enseñanza de las matemáticas con recursos didácticos que incorporen recursos tecnológicos, puede disminuir las dificultades de aprendizaje.

\section{Conclusiones}

En este artículo se han descrito los recursos didácticos y tecnológicos en el aprendizaje de la integral definida, así como el proceso de evaluación, y las dificultades de comprensión. Por tanto, se presentan las conclusiones, tomando como referencia la idoneidad epistémica y ecológica, idoneidad interaccional y mediacional, idoneidad cognitiva y afectiva de un proceso de instrucción matemático (Godino, 2013).

\section{Idoneidad epistémica y ecológica}

El profesorado diseña e implementa recursos didácticos tomando en cuenta el currículo académico de la espacialidad, programas de la asignatura y libros de textos, en un conjunto de acciones que lleven a recrear, crear, diseminar y al intercambio de conocimientos, saberes y prácticas desde la interacción entre el conocimiento local 
ancestral y conocimiento occidental en lo económico, productivo, social, cultural y ambiental que conlleve a la producción de nuevas teorías (URACCAN, 2008). Lo que hace posible la transposición didáctica, es decir, transformar saberes y prácticas de una comunidad en un saber de objeto de enseñanza.

\section{Idoneidad interaccional}

El profesorado planifica el proceso de instrucción de la integral definida considerando aspectos como: la definición del enfoque didáctico; axioma de múltiples experiencias -prácticas, ficticias, hipotéticas-; dilucidación de múltiples ambientes de aprendizaje; ilustración de ambientes de aprendizaje para la diversidad; axioma de la utilización de recursos tecnológicos con participación activa y propositiva; enunciación del propósito de las matemáticas en la especialidad; y que los recursos didácticos estén acorde al planteamiento de la temática. En este sentido, la enseñanza de la integral definida se configura como un proceso de negociabilidad de lo matemático en una comunidad de práctica (León, et al., 2013). Por tales razones, el proceso de evaluación es asumida desde la perspectiva de comunidad de práctica, el profesorado propone: estimulación para la participación, dominio del grupo, dominio de la disciplina, instrumentos de evaluación, estilos metodológicos y la planificación adecuada. Destacando que el trabajo en construcción de los aprendizajes en comunidad de práctica se realiza a partir de los grupos interactivos con énfasis en el reconocimiento de la diversidad de los participantes y una comunicación horizontal.

\section{Idoneidad mediacional}

El profesorado de matemáticas toma como referencia los campos estructurantes personal-familiar, social-comunitario, profesional-especializado, y científico-tecnológico (Rico, 2006; Goñi, 2008), para incorporar al proceso de enseñanza, recursos tecnológicos como la plataforma virtual Moodle, software matemáticos y dispositivos tecnológicos, convirtiéndolos en materiales didácticos y tecnológicos para el aula de clases, entre ellos: syllabus, unidades didácticas, guías de aprendizaje, objetos virtuales de aprendizaje, modelización de las situaciones matemáticas, calculadoras, ordenadores y celulares. Con la finalidad de interactuar con el estudiantado en situaciones didácticas en el contexto social y cultural del estudiantado a través de Power Point, Excel, videos tutoriales, pizarra interactiva, periódicos con situaciones económicas, mundos virtuales, a su vez, modelar el concepto y propiedades de la integral definida en situaciones vinculadas a la especialidad, por ejemplo: Costo marginal, ingreso marginal, función oferta, función demanda, superávit de consumidores y productores, inventario diario promedio y el valor presente de un flujo de ingreso. Todo ello, simulado en ambiente pluritecnológicos con aplicaciones informáticas como Maple, Matlab, Wrmaxima, Derive, Mathematics, GeoGebra. 


\section{Idoneidad cognitiva y afectiva}

El análisis detallado en las dificultades de comprensión en el aprendizaje de la integral definida, se encontró que el estudiantado posee los conocimientos previos para estudiar la integral definida; sin embargo, el análisis del cuestionario cognitivo explica ciertas dificultades asociadas al álgebra, obstáculos cognitivos y errores en el proceso de generalización. Por tales razones, el profesorado incorpora sistemas de prácticas compartidas, para evaluar el aprendizaje en términos de grupos interactivos y actuaciones dialógicas con la finalidad de resolver problemas contextualizados. También se encontró que, el papel afectivo hacia las matemáticas juega un papel importante en el aprendizaje de la integral definida, partiendo de la idea, que la resolución de cualquier problema matemático lleva asociada una situación afectiva para el sujeto implicado, quien pone en juego no solamente prácticas operativas y discursivas para dar respuesta al problema, sino también moviliza creencias, actitudes, emociones o valores que condicionan en mayor o menor grado y diferente sentido la respuesta cognitiva (Godino, 2013). Con referencia a lo anterior, se puede expresar que, el estudiantado está motivado en aprender conceptos y propiedades de la integral definida relacionado con su especialidad de estudio en el sentido que las explicaciones del profesorado le dan confianza para posteriormente resolver las tareas o situaciones matemáticas, además que les motiva y agrada interactuar con recursos didácticos y tecnológicos que el profesorado incorpora en el aula de clases.

La idoneidad didáctica de un proceso de instrucción matemática nos permitió aclarar las ideas expresadas por el estudiantado y el profesorado en el proceso de enseñanza y aprendizaje de la integral definida. Por último, el estudio permite concluir que la incorporación de recursos didácticos y tecnológicos en la enseñanza de la integral definida disminuye las dificultades de comprensión en el aprendizaje del estudiantado, lo que se traduce en que al diseñar e implementar recursos didácticos y tecnológicos, se convierte en un entorno de agrado, motivación y confianza para que el estudiantado sea capaz de resolver tareas matemáticas, y por tanto, disminuir sus dificultades de aprendizaje.

\section{Lista de referencias}

Alonso, F., Barbero, C., Fuentes, I., Azcárate. M., Dozagarat, J., Gutiérrez, S., Ortíz, A., Rivière, V., \& Veiga, C. (1993). Ideas y actividades para enseñar álgebra. Madrid: Editorial Síntesis.

Cardoso, E., Vanegas, E., \& Cerecedo, M. (2012). Diagnóstico sobre las actitudes hacia las matemáticas del estudiantado que inicia sus estudios en tres posgrados en Administración de Empresa. Revista Electrónica Educare, 16(2), 237-253. 
Coll, C. \& Colomina, R. (1991). Interacción entre alumnos y aprendizaje escolar. En C. Coll, J. Palacios, \& A. Marchesi (Eds.). Desarrollo psicológico y educación, II, Psicología de la Educación (335-352). Madrid: Alianza Editorial.

Duval, R. (1995). Sémiosis et Penseé Humaine. Registres sémiotiques et apprentissages intellectuels. Neuchatel: Peter Lang.

Flores, W., O., \& López, E. (2010). Propuesta metodológica en la enseñanza de la integral definida utilizando entornos informáticos para la carrera de Administración de Empresas. Nicaragua: URACCAN.

Flores, W., O., \& Salinas, M. (2013). Metodologías en la enseñanza de la derivada: URACCAN-Nueva Guinea. Ciencia e Interculturalidad, 12(1), 39-49.

Flores, W., O., \& Rojas, N. (2013). Integración de las Tecnologías de la Información y Comunicación en la Enseñanza Universitaria. Horizontes Pedagógicos, 15(1), 21-33.

Flores, W., O., \& Auzmendi, E. (2015). Análisis de la estructura factorial de una escala de actitud hacia las matemáticas. Aula de Encuentros, 17(1), 45-77.

Flores, W., O. (2015a). La formación de profesores de matemática desde el ámbito de la universidad comunitaria intercultural. Ciencia e Interculturalidad, 16(1), 32-53.

Flores, W., O. (2015b). Los problemas asociados a la comprensión del álgebra en estudiantes universitarios. Horizontes Pedagógicos, 17(1), 8-23.

Flores, W., O. (2016). Análisis ontosemiótico en los procesos de resolución de problemas matemáticos por estudiantes universitarios. Bilbao: Universidad de Deusto.

Gil, D., Sáiz, M., Cavanzo, A., León, O., Medina, R., Bonilla, M., Romero, J., Correal, M., Flores, W., O., Rojas, N., Peralta, M., Baca, J., Ávila, C., \& Márquez, A. (2013). Una experiencia de construcción de recursos educativos abiertos, para la formación de profesores de matemáticas en contexto diversidad en el proyecto ALTER-NATIVA. En A. Moreira, L. Bengochea, \& J. Hilera (Eds.). Congreso Internacional para una formación virtual accesible y de calidad (170-177). Lisboa: Universidad de Lisboa.

Godino, J., D. (2013). Indicadores de la idoneidad didáctica de procesos de enseñanza y aprendizaje de las matemáticas. Cuadernos de Investigación y Formación en Educación Matemática, 11, 111-132.

Goñi, J., M. (2008). El desarrollo de la competencia matemática. España: Grao.

González-Martín, A. (2002). Dificultades, obstáculos y errores en el aprendizaje del concepto de integral impropia. Laguna: Universidad de Laguna. 
González-Martín, A., \& Camacho, M. (2005). Sobre la comprensión en estudiantes de matemáticas del concepto de integral impropia. Algunas dificultades, obstáculos y errores. Enseñanza de las Ciencias, 23(1), 81-96.

Mandler, G. (1989). Affect and Learning: Causes and Consequences of Emotional Interactions. In D. McLeod \& V. Adams (Eds.). Affect and Mathematical Problem Solving: A new Perspective (3-19). New York: Springer-Verlag.

León, O., Medina, R., Sáiz, M., Bonilla, M., Romero, J., Gil, D., Correal, M., Flores, W., O., Rojas, N., Peralta, M., Cavanzo, A., Baca, J., Ávila, C., \& Márquez, A. (2013). Relaciones entre "Diseño para Todos" y "Diseño con Todos" en Formación de Profesores de Matemáticas. En A. Moreira, L. Bengochea, \& J. Hilera (Eds.). Congreso Internacional para una formación virtual accesible y de calidad (162169). Lisboa: Universidad de Lisboa.

León, O., Bonilla, M., Romero, J., Gil, D., Correal, M., Ávila, C., C., Bacca, J., Cavanzo, A., Guevara, C., Sáiz, M., García, R., Saíz, E., Rojas, N., Peralta, M., Flores, W., O., \& Márquez, H. (2014). Referentes Curriculares con Incorporación de Tecnologías para la Formación del Profesorado de Matemática en y para la Diversidad. Bogotá: Universidad Distrital Francisco José de Caldas.

Molina, M., Castro, E., \& Castro, E. (2009). Elementary Students' Understanding of the Equal Sign in Number Sentece. Electronic Journal of Research in Educational Psycholog, 7(1), 341-368.

Rico, L. (2006). Marco teórico de evaluación en PISA sobre matemáticas y resolución de problemas, Educación Matemática, Extraordinario, 275-294.

Stake, R. (1999). Investigación con estudio de caso. Madrid: Morata.

URACCAN (2008). Proyecto Educativo Institucional de la URACCAN. Nicaragua: URACCAN.

URACCAN (2014). Plan Estratégico Institucional 2015-2019. Nicaragua: URACCAN.

Wenger, E. (2001). Comunidades de Práctica. Aprendizaje, significado e identidad. Buenos Aires: Paidós. 